\title{
Novel water soluble dendrimer nanocarrier for enhanced photodynamic efficacy of protoporphyrin IX
}

\author{
Murugesan Suresh $\mathrm{Kumar}^{1}$, Anish Babu ${ }^{2}$, Ramachandran Murugesan ${ }^{2,3}$, Kadarkaraithangam Jeyasubramanian ${ }^{1}$ * \\ ${ }^{1}$ Department of Nanoscience and Technology, Mepco Schlenk Engineering College, Sivakasi-626005, INDIA \\ ${ }^{2}$ School of Biological Sciences, Madurai Kamaraj University, Madurai-625021, INDIA \\ ${ }^{3}$ Facuty of Allied Health Sciences, Chettinad Academy of Research and Education, Kelambakkam, Chennai-600310, INDIA
}

*Corresponding author: kjeya@mepcoeng.ac.in; kjeyasubramanian@yahoo.co.in

Tel/fax.: 919486028170

\begin{abstract}
The present study describes the development and evaluation of a novel biocompatible dendrimer-based nano drug delivery system which is readily soluble in water prepared by condensing Phloroglucinol and Succinic Acid (PGSA) and could efficiently encapsulate a well known hydrophobic photodynamic therapy (PDT) agent, protoporphyrin IX (PpIX). The (dark and photo) cytotoxicity of the PGSA-PpIX (dendrimer-drug) formulation towards Dalton Lymphoma Ascites (DLA) cancer cell lines upon visible light treatment is reported and evaluated the cytotoxic Reactive Oxygen Species (ROS) generation efficiency of the Protoporphyrin IX in free and dendrimer encapsulated forms. The in vitro toxicity demonstrated by PpIX loaded PGSA dendrimer nanoformulation, on DLA cells reveals that this novel PGSA nanocarrier reduces the toxic nature of PpIX when compared to free PpIX without light treatment which satisfy the aim of our study. On the other hand, treatment of DLA cells with PGSA dendrimer formulation in combination with light resulted in significant enhancement in therapeutic efficacy of the PDT agent, PpIX.
\end{abstract}

Keywords: Dendrimer, Phloroglucinol-Succinic acid, nano carrier, PpIX, ROS generation, in vitro toxicity, Photodynamic Therapy

Citation: M.S. Kumar, et al. Novel Water Soluble Dendrimer Nanocarrier for Enhanced Photodynamic Efficacy of Protoporphyrin IX. Nano Biomed. Eng. 2012, 4(3), 132-138. DOI: $10.5101 /$ nbe.v4i3.p132-138.

\section{Introduction}

Photodynamic therapy (PDT) in cancer treatment involves the uptake of photosensitizer by cancer tissue followed by photoirradiation [1]. Since photosensitizers are acting selectively in the illuminated area, they produce lesser adverse effect especially in cancer treatment [2]. The combined action of an excited photosensitizer and molecular oxygen results in the formation of singlet oxygen $\left({ }^{1} \mathrm{O}_{2}\right)$, which is thought to be the main mediator of cellular death introduced by PDT [3]. By PDT body's immune response against tumor can also be activated in addition to direct killing of tumor cells $[4,5]$.

Most photosensitizers are highly hydrophobic; an effective interaction is possible if the delivery system is also the same hydrophobic type. The essential property of drug delivery system is, it could be biodegradable, minimum immunogenicity, incorporate the photosensitizer without loss or alteration of its activity and provide an environment where the photosensitizer can be administered in monomeric form (reducing aggregation which can decrease singlet oxygen quantum yields) [6]. Different approaches have been proposed to incorporate the PS in delivery systems like liposomes, micelles, polymeric particles, low density lipoproteins, etc. [7].

However, unlike other drugs, photosensitizers are not by themselves toxic to the targeted cells rather they act like catalyst to create toxic products from non-toxic dissolved oxygen, so that they can be used repeatedly with proper activation. In such a scenario, biocompatible carriers can be used as carriers to evade problems of usage of free (dark toxic) drugs [3]. One of the major problem reported clinically for PS in PDT is, its side effects to normal tissue by dark toxicity, so the usefulness of a PS can be made possible by its minimal dark toxicity $[8,9]$. 
The use of nanoparticles as carriers of photosensitizers is becoming a very promising approach which can satisfy all the requirements of an ideal PDT agent [1]. Moreover nanoparticle based approach makes favour for the PS to deliver it more selectively at the tumor site, by reducing its toxicity and damage to the healthy tissues [10]. Nanoparticles carrying PS by different strategies (adsorption/covalent bonding) offer benefits of hydrophilicity and appropriate size for passive targeting to tumour tissues by the enhanced permeability and retention (EPR) effect [3].

Dendrimer, as a nano drug delivery system, offers a uniform and promising route for drug entrapment, conjugation, and controlled release [11]. Few reports have been published so far on designing dendrimers containing various photosensitizers for the formation of singlet oxygen in the tumor tissue [12]. Dendrimers with carboxylate (or) hydroxyl groups on its surface, for example glycodendrimers [13] and Poly(propyl ether imine) have been demonstrated as biocompatible nano drug delivery vehicles for anti-cancer drugs [14-16].

Biodegradable polymeric nanocarrier namely chitosan nanoparticles(CNPs) having an advantage of incorporating the hydrophobic drug PpIX to its hydrophobic inner core showed an improved tumor specificity and therapeutic efficiency for photodynamic therapy compared to free PpIX [17]. Studies on photosensitizers like PpIX, Rose Bengal (RB) with PAMAM and PPI dendrimers in HeLa cell lines, and with cyclic peptides in human cervical cancer SiHa cell line [18] are revealed to be non-toxic by PEGylation on their surface and also had good photodynamic activity both in vitro and in vivo [19]. By improving the properties of dendrimer through appropriate unfunctionalization of their periphery and tailoring to therapeutic needs makes it as a promising carrier for photosensitizers.

However, the cationic dendrimers produce cytotoxicity because of the negatively charged cell membrane and the positively charged dendrimer surface, which enable these dendrimers to adhere and damage the cell membrane, causing cell lysis $[12,20]$. As revealed by Malik et al. [21], regardless of structure, cationic dendrimers were hemolytic and cytotoxic even at relatively low concentration and the biocompatibility or safety of dendrimers in vivo is dominated by its charge. Conversely, dendrimers with carboxylate-terminal groups were neither hemolytic nor did they cause cytotoxicity across a panel of cell lines studied in vitro [20].

Biocompatibility of the most PAMAM, Poly(lysine) dendrimers with cytotoxic (cationic) surface charged groups are improved to anionic surface with the aid of PEGylation, anionic amino acid such as glutamate over their cationic surface functionalities [22-24]. Despite successful talk on drug encapsulation and the biocompatibility of dendrimers, the fate of dendrimer is to readily degrade from the body as a non-toxic product after its administration [25]. Many of the reports have been reported the problem of cytotoxicity with amine and ammonium terminated cationic dendrimers and affirmed that when compared to cationic surface functionality dendrimers, anionic (carboxyl and hydroxyl terminated) dendrimers are believed to be non-toxic on primary cell lines [26,27].

So the charge property plays a vital role in determining the dendrimer cytotoxicity and moreover commercially available dendrimers functionalized with PEGylation, Acetylation, and glycosylation has been reported to be biocompatible for nano drug delivery with minimal cytotoxicity and nanotoxicity [25]. The only tactic to lower the toxicity problem on dendrimers is to improve its surface functionalities to be biocompatible [22,28,29]. In this paper, a novel and water soluble biocombatible anionic dendrimer was fabricated by reacting 2,4,6-trihydroxy benzene (Phloroglucinol) with succinic acid as precursors in suitable solvent and established as a novel drug delivery system for PpIX, a PDT agent for its enhanced PDT efficacy in model DLA cell lines. The novel dendrimer possessing anionic surface functional groups was physiochemically characterized for PDT. In addition to this, drug loading efficiency, in vitro drug release, reactive oxygen species (ROS) efficiency were also determined. Further, the toxicity of the novel dendrimer formulation of PpIX was evaluated in DLA cells in vitro.

\section{Experimental section}

\subsection{Materials}

Phloroglucinol (2,4,6 trihydroxy benzene) and Succinic acid were purchased from Kemphasol, Bombay, India. Protoporphyrin IX was purchased from Spectrum chemicals, USA. Millipore water was used throughout the study. All the other chemicals were used as they received.

\section{Methods:}

The chemical transformation performed at the surface of dendrimers, its respective generations and the association of the drug to dendrimer were scrutinized using Fourier transform infrared spectroscopy (FT-IR, Bruker optics GmbH-Alpha T spectrometer, Germany). ${ }^{1} \mathrm{H}$ NMR spectra of half (2.5) and full generation (3.0) dendrimers in $\mathrm{CDCl}_{3}$ solvent were recorded using 300 MHz NMR (BRUKER, Germany) spectroscopy.The surface charge (zeta potential) of the PGSA dendrimers were measured using Zeta sizer (Malvern instrument, UK) and the surface morphology of the dendrimers were examined using atomic force microscopy in noncontact mode (XE 70, SPM, Park system, South Korea). Uv-Vis. Spectroscopy of the dendrimers and the drug etc., in various solvents were measured in Perkin-Elmer Lambda-25 spectrophotometer.

\subsection{Synthesis of biocompatible PGSA dendrimer and PpIX encapsulated PGSA dendrimer}




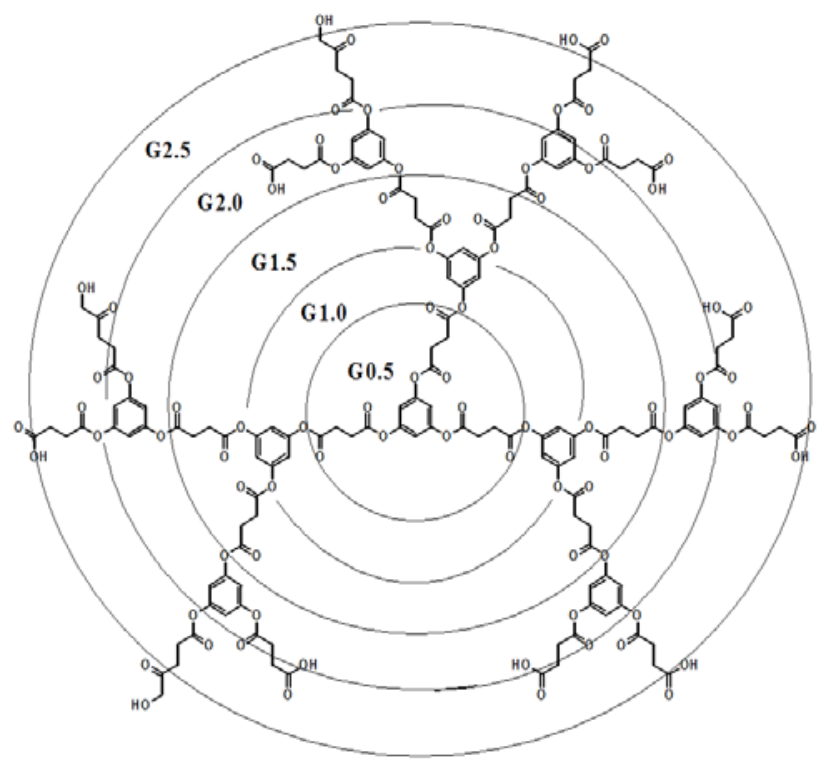

Fig. 1 Structure of G2.5 dendrimer

$1.28 \mathrm{~g}(0.01 \mathrm{M})$ of phloroglucinol (2,4,6-trihydroxybenzene) and $3.546 \mathrm{~g}(0.03 \mathrm{M})$ of succinic acid were dissolved in $125 \mathrm{~mL}$ of water. 2-3 drops conc. $\mathrm{H}_{2} \mathrm{SO}_{4}$ was added as catalyst to initiate the esterfication reaction and refluxed for 10 hours at $60{ }^{\circ} \mathrm{C}$. After refluxing, the solvent was reduced to one third of its original volume using rotary evaporator and the contents were kept at $0{ }^{\circ} \mathrm{C}$ for about 24 hours. The G0.5 dendrimer in crystallized form was filtered and air dried. Since the core group (G0.5) possess three carboxylic acid end groups, it can further reacts with another 3 molecules of phloroglucinol which yields G1.0 dendrimer. The same reaction procedure was followed for synthesizing G1.5, G2.0, G2.5, and G3.0 biocompatible dendrimers with proper molar ratios of phloroglucinol and succinic acid (The average molar ratio was calculated on the basis of average molecular weight and average number of end groups in each generation). The predicted G2.5 dendrimer structure is shown in Fig . 1.

\section{Loading of photosensitizer into the dendrimer:}

$250 \mathrm{mg}$ of G2.5 dendrimer was dissolved in $20 \mathrm{~mL}$ of water. To this dendrimer solution, protoporphyrin IX ( $5 \mathrm{mg}$ in $2 \mathrm{~mL}$ acetonitrile) was added drop-wise and stirred vigorously by keeping over magnetic stirrer for two hours. Water was then removed by using rotary evaporator. The solid particles were then filtered and used for further analysis.

\subsection{Drug loading and encapsulation efficiency}

The drug encapsulated dendrimer was dissolved in acetonitrile and the absorbance was measured at 400 $\mathrm{nm}$ the absorption maxima of PpIX using UV-Vis. spectrophotometer. From the absorbance of the drug, the concentration of encapsulated PpIX has been determined from a standard graph. The drug loading (DL) and encapsulation efficiencies (EE) were calculated using the following formulae:

Drug loading $=\left(\mathrm{W}_{1} / \mathrm{W}_{2}\right) \times 100 \%$

Encapsulation efficiency $=\left[\left(\mathrm{W}_{3}-\mathrm{W}_{4}\right) / \mathrm{W}_{3}\right] \times 100 \%$

Here, $\mathrm{W}_{1}$ is the weight of the drug encapsulated; $\mathrm{W}_{2}$ is the gross weight of the dendrimer; $\mathrm{W}_{3}$ is the total weight of the drug added initially; $\mathrm{W}_{4}$ is the weight of the drug released in the liquid medium [30].

\subsection{In Vitro Release kinetics studies}

The in vitro drug release experiment was carried out using the dialysis tube diffusion technique [31]. The in vitro experiments were performed in phosphate buffer saline (PBS) at $37{ }^{\circ} \mathrm{C}$ under sink conditions. Drug loaded dendrimer of $1 \mathrm{mg} \mathrm{mL}^{-1}(5 \mathrm{~mL})$ was taken in a dialysis bag (Himedia, India) with a 12000-14000 Da ( 2.4 $\mathrm{nm})$ cut-off pore size and immersed in $20 \mathrm{~mL}$ of PBS containing $200 \mu \mathrm{L}$ of DMSO (pH 7.2) in a beaker with constant stirring. Samples were withdrawn periodically and replaced with fresh PBS and the amount of the drug was quantified spectrophotometrically at $\lambda=400 \mathrm{~nm}$ from the standard graph. The released drug was calculated in percentage relative to the initial concentration taken for dialysis.

\subsection{Qualitative yield of Reactive Oxygen Species (ROS) generation by iodide method}

The ${ }^{1} \mathrm{O}_{2}$ generation by PpIX and PpIX-dendrimer was investigated by the iodide method, in which the photogenerated ${ }^{1} \mathrm{O}_{2}$ reacts with $\mathrm{I}^{-}$in the presence of $\left(\mathrm{NH}_{4}\right)_{2} \mathrm{MoO}_{4}$ as a catalyst [32]. The reaction product is (followed at $351 \mathrm{~nm}$ using a UV-Vis spectrophotometer), which is $\mathrm{I}_{3}^{-}$directly proportional to the generated ${ }^{1} \mathrm{O}_{2}$. The efficiency of photogeneration of ${ }^{1} \mathrm{O}_{2}$ by PpIX as well as PpIX-dendrimer was determined based on the relative rates of $\mathrm{I}_{3}^{-}$generation, under identical conditions. Briefly, the absorbance of free PpIX or PpIX-dendrimer was randomly selected (random concentrations) so that its value should be less than 0.1 . For each concentration of the test solution, irradiation was performed at equal time intervals. The experimental points for each concentration expressed by the absorbance at $\lambda=351 \mathrm{~nm}$ were plotted against irradiation time in seconds to get a curve.

\subsection{In Vitro Toxicity studies}

Dalton Lymphoma Ascites (DLA) cells were seeded into 96-well plates at a density of approximately $2.5 \times 10^{4}$ cells per well in RPMI 1640 media. Following incubation for $12 \mathrm{~h}$ medium was changed and various concentrations $\left(0.02 \mu \mathrm{g} \mathrm{mL}^{-1}, 0.04 \mu \mathrm{g} \mathrm{mL}^{-1}\right.$ and $\left.0.08 \mu \mathrm{g} \mathrm{mL}^{-1}\right)$ of PpIX in free and dendrimer encapsulated forms were added to their designated wells for $2 \mathrm{~h}$ incubation period. The plates were then irradiated using a $150 \mathrm{~W}$ xenon arc lamp (Photon technology International, US) for 5 minutes $\left(30 \mathrm{~mW} \mathrm{~cm}^{-2}\right)$. Immediately after irradiation, the cells were washed using PBS ( $\mathrm{pH}$ 7.2) to remove free PpIX or dendrimer particles. Phototoxicity assessment was carried 
out using the MTT (3-[4,5-dimethylthiazol-2-yl]-2,5diphenyl-tetrazoliumbromide) cell viability assay based on the activity of mitochondrial dehydrogenases. After the treatment, MTT solution was added to each well in a concentration of $5 \mathrm{mg} \mathrm{mL}^{-1}$, and the plates were incubated at $37{ }^{\circ} \mathrm{C}$ for 4 hours. The resulting formazan crystals were dissolved by the addition of $100 \mu \mathrm{L}$ of isoproponol after removing the medium. Plates were read at $570 \mathrm{~nm}$ (MR 700 Dynatech, UK) and the mean cell survival was calculated for free PpIX and PGSA-PpIX formulations for each concentration and expressed as a percentage of control (cells not irradiated) cell survival values. For the determination of dark toxicity of the compounds, similar experiments were carried out but without irradiation.

\section{RESULTS AND DISCUSSION}

\subsection{Characterization of the dendrimer formula- tion}

\subsubsection{FT-IR spectroscopy}

The formation of G0.5, G1.0, G1.5, etc., dendrimer was confirmed from their FTIR spectra. In all stages, the dendrimer has been formed as result of esterfication of acidic $-\mathrm{COOH}$ group originating from succinic acid and the phenolic $-\mathrm{OH}$ group from phloroglucinol. The Fig. 2 represents the FTIR spectra of a) Phloroglucinol b) Succinic acid c) G0.5 and d) G2.0. The precursor phloroglucinol show a prominent peak at $3191 \mathrm{~cm}^{-1}$ characteristic for phenolic $-\mathrm{OH}$ group and the succinic acid shows a characteristic - $\mathrm{COOH}$ group peak at 1680 $\mathrm{cm}^{-1}$. On esterfication, the phenolic $-\mathrm{OH}$ group gets condensed with the $-\mathrm{COOH}$ group of the succinic acid. By examining the IR spectra (Fig. 2c), it is clear that the formed product was an ester of G0.5 core dendrimer because, the disappearance of the peak at $3191 \mathrm{~cm}^{-1}$ is observed with the shift in peak position of acidic $-\mathrm{COOH}$ group into the ester group of $1688 \mathrm{~cm}^{-1}$. The IR spectra of full generation dendrimer (G2.0- Fig. 2d) exhibit an additional peak around $3154 \mathrm{~cm}^{-1}$ characteristic for the phenolic $-\mathrm{OH}$ end group. Similar results were obtained for all half generation dendrimer (G0.5, G1.5, G2.5) with an ester peak around $1600-1650 \mathrm{~cm}^{-1}$ and peaks around $3100 \mathrm{~cm}^{-1}$ for full generation dendrimer $(\mathrm{G} 1.0, \mathrm{G} 2.0$, G3.0).

The encapsulation of drug by dendrimer is also confirmed from their corresponding IR spectra of pure drug (Fig. 3b), the drug loaded G3.0 dendrimer (Fig. 3c) and the pure dendrimer (Fig. 3a). The characteristic absorption peak at $3169 \mathrm{~cm}^{-1}$ is observed for the phenolic - OH group (Fig. 3c) which is the end group on the G3.0 dendrimer surface, then the spectra of pure PpIX (Fig. 3b) shows a sharp stretching vibration at $1699 \mathrm{~cm}^{-1}$ for the carboxylic acid functional group. The PpIX loaded G3.0 dendrimer (Fig. 3a) exhibit the strong absorption peak at $3146 \mathrm{~cm}^{-1}$ for the phenolic - $\mathrm{OH}$ group and the carboxylic acid group of PpIX at $1685 \mathrm{~cm}^{-1}$ indicates the existence of both the drug and the dendrimer as a host-guest matrix.

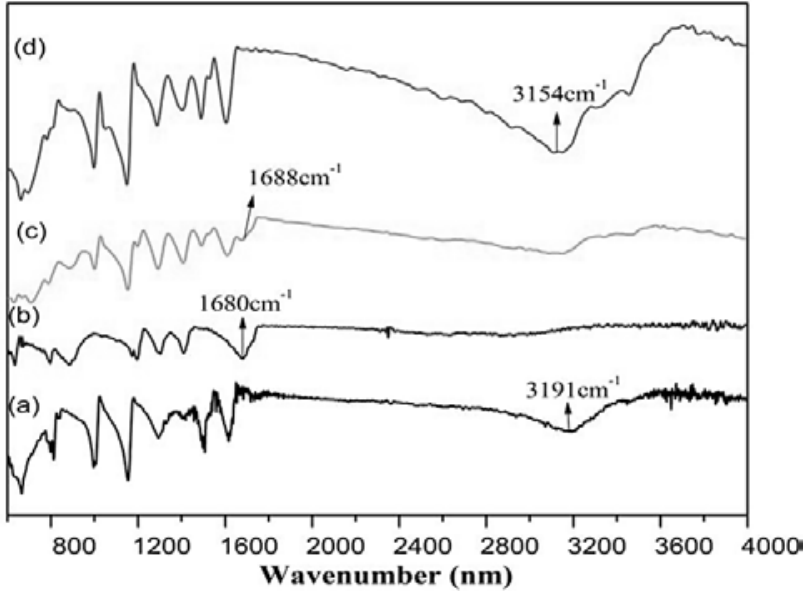

Fig. 2 FTIR Spectra of a) Phloroglucinol b)Succinic acid c) 0.5 Generation d) 2.0 Generation dendrimer.

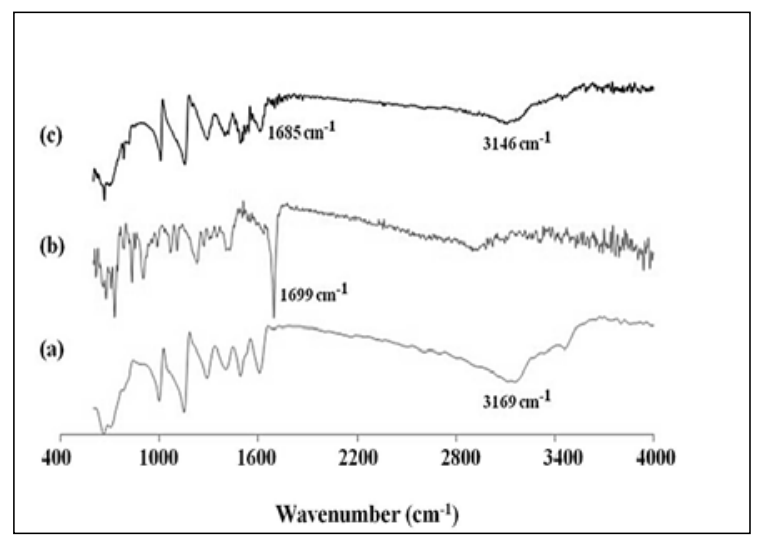

Fig. 3 FTIR spectra of (a) G3.0 dendrimer (b) PpIX (c) PpIX encapsulated G3.0 dendrimer

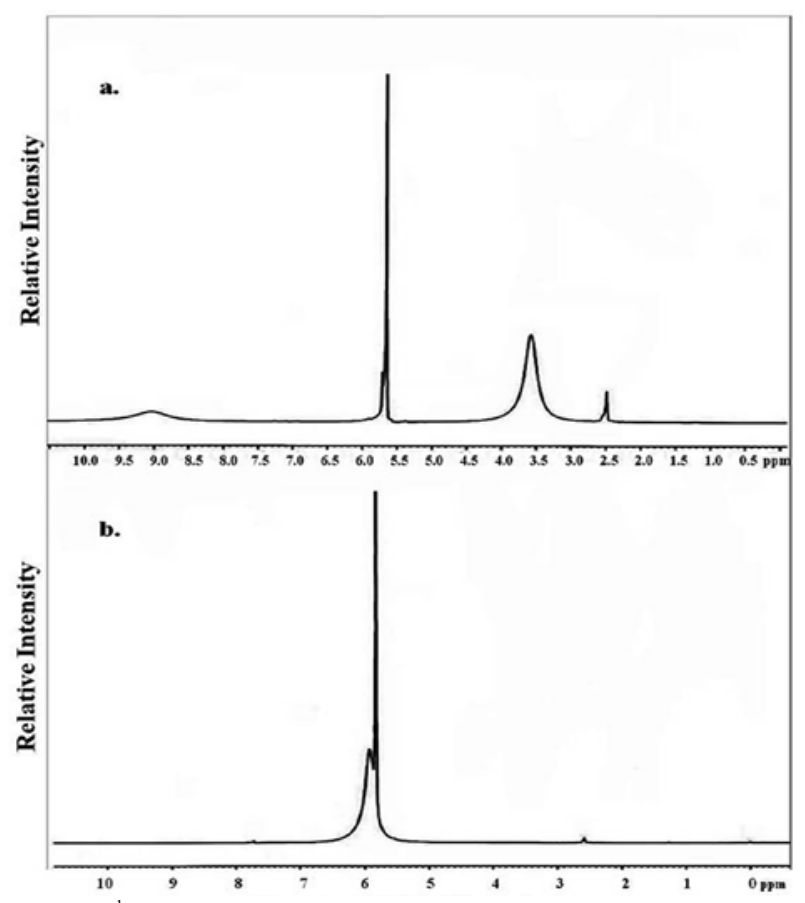

Fig. $4{ }^{1} \mathrm{H}$ NMR spectra of a) G2.5 dendrimer and b) G3.0 dendrimer 


\subsubsection{NMR spectroscopy}

Fig. 4 represents the NMR signals of a half $(\mathrm{G} 2.5)$ [Fig. 4a] and full (G3.0) [Fig. 4b] generation dendrimer recorded in $\mathrm{CDCl}_{3}$ solvent. The peak at 6-7 $\delta \mathrm{ppm}$ is assigned to aromatic proton, $2-3 \delta \mathrm{ppm}$ is assigned to $\mathrm{CH}_{2}$ proton of succinic acid and the appearance of a weak signal is assigned to $-\mathrm{OH}$ proton at 9-12 $\delta \mathrm{ppm}$. The ${ }^{1} \mathrm{H}$ NMR of G2.5 of PGSA represented in Fig. 4a shows the signals at $9.04 \delta \mathrm{ppm}, 5.64 \delta \mathrm{ppm}$, and $2.482 \delta$ ppm for the presence of methyl proton and $\mathrm{OH}$ proton of succinic acid, and an aromatic proton of phloroglucinol respectively.

\subsubsection{Particle size and Zeta potential measurements}

Fig. 5a and Fig. 5b represents the 2D and 3D AFM images of G2.5 dendrimer and gives information about the particle size distribution. The nanoparticles in the scanned region posses near-spherical shape with an average particle size of $120 \mathrm{~nm}$.

The zeta potential values of dendrimers of various generations are given in Table 1. Both the half generation and full generation dendrimers showed negative potential values because of the presence of carboxylic acid and phenolic hydroxyl groups as their terminal surface groups (both are anionic), which can be easily ionised in an aqueous medium.

\subsection{Optical properties of protoporphyrin IX encapsulated G2.5 dendrimer}

The UV-visible absorption spectra of G2.5 dendrimer and the drug loaded dendrimer in aqueous solution is

(a)

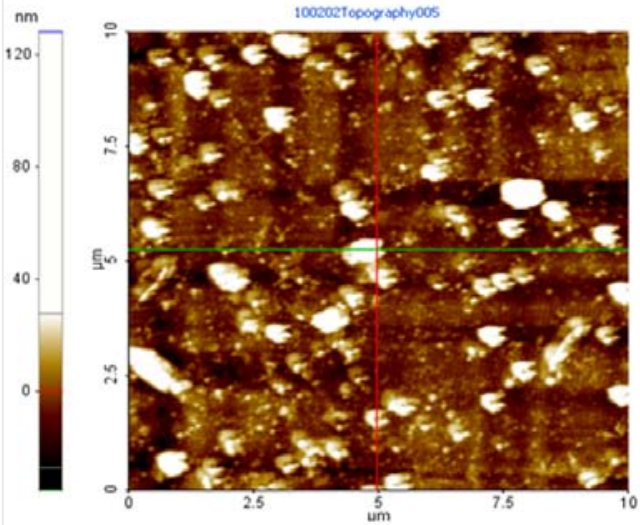

(b)

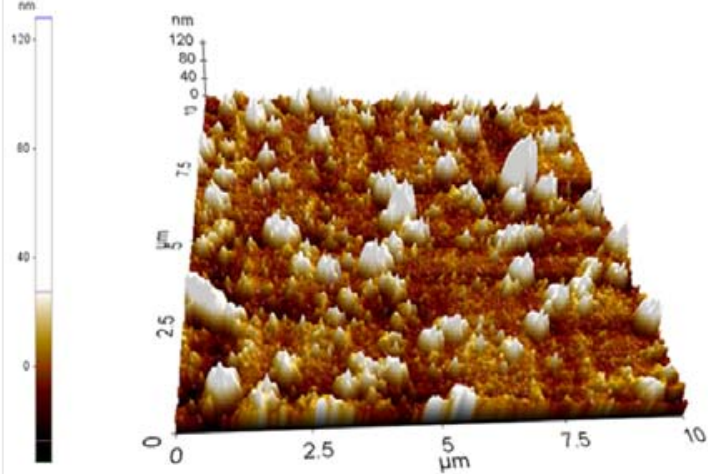

Fig. 5 2D (a) and 3D (b) AFM image of G2.5 dendrimer
Table 1 Zeta potenital of various generations of PGSA dendrimers

\begin{tabular}{|l|l|}
\hline Generation of dendrimers & Zeta potential $(\mathrm{mV})$ \\
\hline G1.0 & -29.7 \\
\hline $\mathrm{G} 1.5$ & -22.9 \\
\hline $\mathrm{G} 2.0$ & -23.2 \\
\hline $\mathrm{G} 2.5$ & -34.0 \\
\hline
\end{tabular}

shown in Fig. 6. The UV-visible absorption spectra of G2.5 dendrimer in figure (as dashed line) shows, its maximum absorption in the region around $230 \mathrm{~nm}$ and $440 \mathrm{~nm}$. The absorption spectrum of the drug loaded dendrimer (dotted line) shows two prominent peaks at $240 \mathrm{~nm}$ and $400 \mathrm{~nm}$. In addition to this, a small hump is also found near $440 \mathrm{~nm}$. All these bands are the combined peaks of both dendrimer and PpIX. The absorption spectrum of pure PpIX in acetonitrile exhibit a sharp peak in the region $400 \mathrm{~nm}$ (solid line) with the absorbance of approximately 1.8. After loading the $\operatorname{drug}(\mathrm{PpIX})$ into the dendrimer, the intensity at $400 \mathrm{~nm}$ (dotted line) is getting decreased (around 0.5). This shows the encapsulation of the drug by the dendrimer.

\subsection{Drug loading and encapsulation efficiency}

The maximum quantity of the drug capturing power of the dendrimer has been evaluated from the drug loading studies which were followed spectrometrically. From the standard graph, the drug encapsulation efficiency (EE) and the drug loading capability (DL) of the dendrimer was calculated to be $96.2 \%$ and $2.9 \%$ respectively.

\subsection{In Vitro release kinetics studies}

The controlled agitation of the drug loaded dendrimer, released the drug from the dendritic cavity in a controlled manner into the PBS medium through the cut off pore size of the dialysis membrane. The quantity of drug released with time has been analyzed spectrophotometrically. Fig. 7 shows the in vitro drug release profile of the PpIX encapsulated G2.5 dendrimer in PBS in the first $60 \mathrm{~h}$. It is clear from the figure that the rate of release of drug is rapid initially during the first $12 \mathrm{~h}$. The drug release from the medium is further enhanced to $24 \%$ within next 24 h. More or less the same quantity of the drug has been released from the system in a sustained manner, once it crosses the initial $12 \mathrm{~h}$ time. The initial rapid release may be due to the release of drug molecules that were weakly interacted with the hydrophilic moiety of the dendrimer

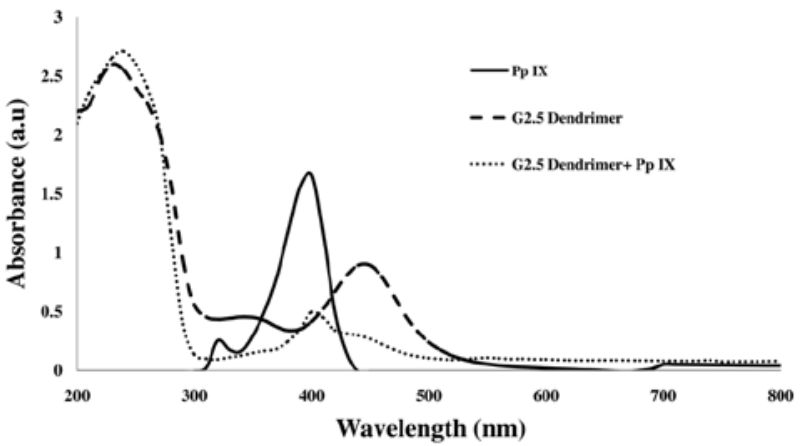

Fig. 6 UV-Vis absorption Spectra of PpIX, G2.5 Dendrimer, PpIX encapsulated G2.5 dendrimer 


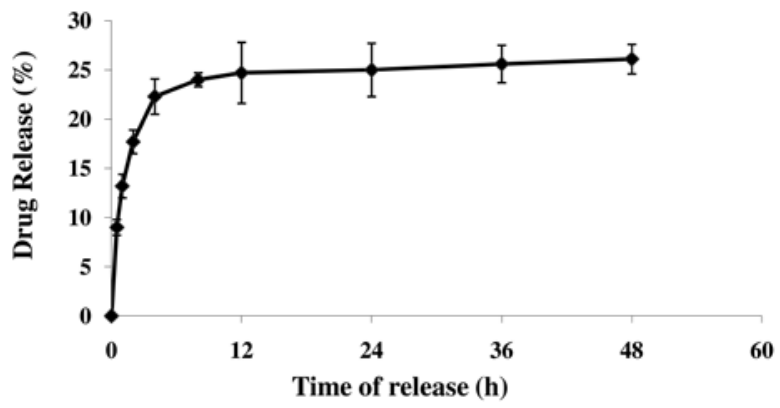

Fig. 7 In vitro Drug Release profile of PpIX encapsulated G2.5 dendrimers

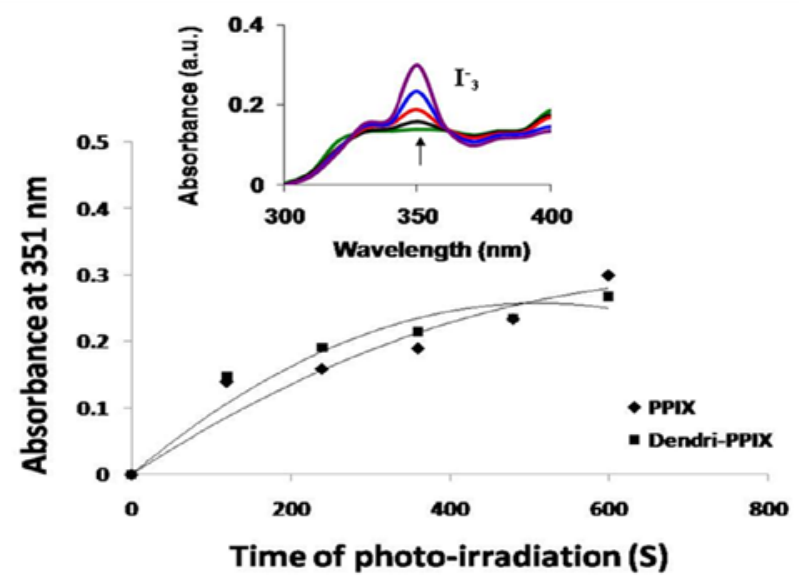

Fig. 8 Qualitative yield of ${ }^{1} \mathrm{O}_{2}$ for free PpIX and G2.5 dendrimerPpIX formulation nanoparticles.

\subsection{ROS Generation efficiency}

The Photogeneration of $\operatorname{ROS}\left({ }^{1} \mathrm{O}_{2}\right)$ by the PpIXdendrimer was determined by the iodide method. Fig. 8 depicts the graphical representation of the change in absorbance of the $I_{3}^{-}$band $(351 \mathrm{~nm})$ against the irradiation time for free PpIX and G2.5 dendrimerPpIX formulations. It is observed that the absorbance increases with photo irradiation time indicative of active singlet oxygen generation. Hence the PpIX encapsulated dendritic system indicates that PpIX remained its ${ }^{1} \mathrm{O}_{2}$ production capability even when it is associated with the dendrimer particles by encapsulation.

\subsection{In Vitro Toxicity studies}

The cytotoxicity of free and dendrimer encapsulated PpIX towards DLA cells in vitro in the dark and light conditions are shown in the Fig. 9. The diagram depicts the percentage of cell survival with respect to untreated control cells. The dark toxicity study lacked significance throughout all the PpIX concentrations (free as well as PGSA dendrimer-PpIX) studied. The application of free PpIX and PGSA dendrimer-PpIX nanoparticle in cells without light treatment led only to a partial reduction of viable cells at its maximum concentration studied (cell viability of $70 \%$ and $72 \%$ respectively at $0.08 \mu \mathrm{g} \mathrm{mL}^{-1}$ ). Application of light alone did not influence cell viability. Combining PpIX formulations and photoirradiation resulted in significant enhancement in cell viability reduction compared to dark toxicity studies. At the lowest

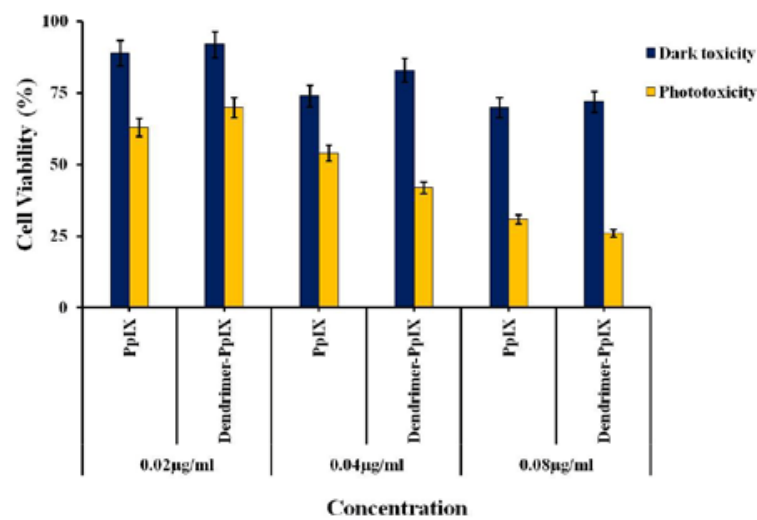

Fig. 9 Cell viability diagram for DLA cells when incubated overnight with free-PpIX and Dendrimer-PpIX as a result of photoirradiation relative to untreated controls. Dark toxicity profile shows the effect of free-PpIX and Dendrimer-PpIX towards DLA cells incubated without photoirradiation.

PpIX concentration studied, PDT resulted in more cell viability for PGSA dendrimer-PpIX formulations (70\%) than free PpIX formulation (63\%). This result indicates that at this concentration $\left(0.02 \mu \mathrm{g} \mathrm{mL}^{-1}\right)$ free PpIX results in more photodynamic effectiveness compared to PGSA dendrimer-PpIX formulation. However, significant differences in phototoxicity were observed between free PpIX and PGSA dendrimer-PpIX on further increase in PpIX concentrations. A gradual decrease in cell viability was observed as $31 \%$ and $26 \%$ for higher concentrations $\left(0.08 \mu \mathrm{g} \mathrm{mL}^{-1}\right)$ respectively between free PpIX and PGSA dendrimer-PpIX formulations. Which means that, the dendrimer prevents the dark toxicity of PpIX at low concentration so kills less number of cells when compared to free PpIX in dark. But after irradiation, the ROS generating capability of G2.5+PpIX was quiet enhanced when compared to free PpIX, and influences the cell death. This result indicates the photodynamic effectiveness of PGSA dendrimer-PpIX formulation against DLA cell lines in vitro and may be attributed to the efficient delivery of PpIX when it is associated with the dendrimer particles.

\section{Conclusion}

The present study reports the synthesis of novel biocompatible dendrimer which is able to load/release the drug in a sustainable manner which is good as a nano drug delivery vehicle especially in PDT of cancer. Dendrimers were characterized using FTIR, UV-Visible spectroscopy, Zeta potential and AFM. Along with PGSA dendrimer, the PpIX retains its ROS generation property upon irradiation and also reduces the toxicity of the drug by encapsulating inside the nano cavities. The present study also highlights the in vitro toxicity of pure drug (free-PpIX) and PGSA dendrimer-PpIX. The dark toxicity of free PpIX is high in comparison with the dendrimer encapsulated drug. The formulation of PpIX with novel dendrimer exhibited efficient cytotoxicity, compared with free PpIX in DLA cell lines. Also, the cytotoxicity was caused by sufficient generation of singlet oxygen during light irradiation. Since we have taken initial steps in this work to ascertain the behavior of our 
novel nano drug delivery system as nano carriers for PS, it is hoped that this may provide useful guidelines for further improvement on delivery of PDT drugs with PGSA dendrimer in solid tumors particularly for PDT of cancer.

\section{Acknowledgements}

The authors wish to thank Mepco Schlenk Engineering College, Sivakasi, TN and Madurai Kamaraj University, TN, India for providing full support and well equipped lab facility for finishing this work successfully.

\section{References}

1 Bechet D, Couleaud P, Frochot C, Viriot ML, Guillemin F, BarberiHeyob M. Nanoparticles as vehicles for delivery of photodynamic therapy agents. Trends Biotechnol. 2008; 26: 612-621. http:// dx.doi: 10.1016/j.tibtech.2008.07.007.

2 Vargas A, Pegaz B, Debefve E, Konan-Kouakou Y, Lange N, Ballini J, van den Bergh H, Gurny R, Delie F. Improved photodynamic activity of porphyrin loaded into nanoparticles: an in vivo evaluation using chick embryos. Int. J. Pharm. 2004; 286: 131-145. http://dx.doi:10. 1016/j.ijpharm.2004.07.029.

3 Dougherty TJ, Gomer CJ, Henderson BW, Jori G, Kessel D, Korbelik M, Moan J, Peng Q. Photodynamic therapy. J. Natl. Cancer Inst. 1998; 90: 889-905.

4 Castano AP, Mroz P, Hamblin MR. Photodynamic therapy and antitumour immunity. Nat. Rev. Cancer 2006; 6(7): 535-545. http://dx. doi:10.1038/nrc1894.

5 Gollnick SO, Brackett CM. Enhancement of anti-tumor immunity by photodynamic therapy. Immunol. Res. 2010; 46: 216-226. http:// dx. doi:10.1007/s12026-009-8119-4.

6 Chatterjee DK, Fong LS, Zhang Y. Nanoparticles in photodynamic therapy: An emerging paradigm. Adv. Drug Delivery Rev. 2008; 60: 1627-1637. http://dx.doi:10.1016/j.addr.2008.08.00.

7 Konan YN, Gurny R, Allemann E. State of the art in the delivery of photosensitizers for photodynamic therapy. $J$. Photochem.Photobiol., B 2002; 66: 89-106.

8 Babu A, Jeyasubramanian K, Gunasekaran P, and Murugesan R. Gelatin nanocarrier enables efficient delivery and phototoxicity of hypocrellin B against a mice tumour model. J. BiomedNanotechnol. 2012; 8: 1-14. http://dx.doi:10.1166/jbn.2012.1354.

9 Roby A, Erdogan S, Torchilin VP. Enhanced in vivo antitumor efficacy of poorly soluble PDT agent, meso-tetraphenylporphine, in PEG-PE-Based tumor-targeted immunomicelles. Cancer Biol. Ther. 2007; 6: 1136-1142.

10 Olivo M, Bhuvaneswari R, Swarnalatha Lucky S, Dendukuri N and Soo-Ping Thong P. Targeted therapy of cancer using photodynamic therapy in combination with multi-faceted anti-tumor modalities. Pharmaceuticals 2010; 3: 1507-1529. http://dx.doi:10.3390/ ph3051507.

11 Qiu LY and Bae YH. Polymer architecture and drug delivery.Pharm Res.2006;23:1-30.http://dx.doi:10.1007/s11095-005-9046-2

12 Boas U and. Heegaard PMH. Dendrimers in drug research. Chem. Soc. Rev. 2004; 33: 43 - 63. http://dx.doi: 10.1039/b309043b.

$13 \mathrm{Li}$ Y, Cheng Y, Xu T. Design, Synthesis and potent pharmaceutical applications of glycodendrimers: A mini review. Current Drug Discovery Technologies, 2007; 4(4): 246-254.

14 Jayamurugan G and Jayaraman N. Synthesis of large generation poly(propyl ether imine) (PETIM) dendrimers. Tetrahedron. 2006; 62: 9582-9588. http://dx.doi:10.1016/j.tet.2006.07.094.

15 Jayamurugan $\mathrm{G}$, Umesh CP, Jayaraman N. Inherent photoluminescence properties of poly(propyl ether imine) dendrimers. Org. Lett. 2008; 10: 9-12. http://dx.doi: 10.1021/ol702635w.

16 Ballico M, Drioli S, and Bonora GM. MultiPEGs: High molecular weight multifunctional poly(ethylene glycol)s assembled by a dendrimer-like approach. Eur. J. Org. Chem. 2005; 2064-2073. http://dx.doi: 10.1002/ejoc.200400704.

17 Lee SJ, Park K, Oh YK, Kwon SH, Her S, Kim IS, Choi K, Lee SG, Kim H, Lee SG, Kim K, Kwon IC. Tumor specificity and therapeutic efficacy of photosensitizer-encapsulated glycol chitosan-based nano- particles in tumor-bearing mice. Biomaterials. 2009; 30: 2929-2939. http://dx.doi:10.1016/j.biomaterials.2009.01.058.

18 Conway CL, Walker I, Bell A, Roberts DJH, Brown SB, Vernon and Vernon DI. In vivo in vitro characteristic of a protoporphyrin IXcyclic RGD peptide conjugate for use in photodynamic therapy. Photochem. Photobiol. Sci. 2008; 7: 290-298. http://dx.doi: 10.1039/ b715141a.

19 Kojima C, Toi Y, Harada A, and Kono K. Preparation of poly(ethylene glycol)-attached dendrimers encapsulating photosensitizers for application to photodynamic therapy. Bioconjugate Chem. 2007; 18: 663-670. http://dx.doi: 10.1021/bc060244u.

20 Wolinsky JB, Grinstaff MW. Therapeutic and diagnostic applications of dendrimers for cancer Treatment. Adv. Drug Delivery Rev. 2008; 60: 1037-1055. http://dx.doi:10.1016/j.addr.2008.02.012.

21 Malik N, Wiwattanapatapee R, Klopsch R, Lorenz K, Frey H, Weener JW, Meijer EW, Paulus W, Duncan R. Dendrimers: Relationship between structure and biocompatibility in vitro, and preliminary studies on the biodistribution of 125I-labelled polyamidoamine dendrimers in vivo. J. Control. Release. 2000; 65: 133-148. http://dx.doi. org/10.1016/S0 168-3659(99)00246-1.

22 Gupta U, Jain NK. Non-polymeric nano-carriers in HIV/AIDS drug delivery and targeting. Adv. Drug Delivery Rev. 2010; 62: 478-490. http://dx.doi:10.1016/j.addr.2009.11.018.

23 Miyano T, Wijagkanalan W, Kawakami S, Yamashita F and Hashida M. Anionic amino acid dendrimer-trastuzumab conjugates for specific internalization in HER2-positive cancer cells. Mol. Pharmaceutics. 2010; 7: 1318-1327. http://dx.doi: 10.1021/mp100105c.

24 Bai S and Ahsan F. Synthesis and evaluation of pegylated dendrimeric nanocarrier for pulmonary delivery of low molecular weight heparin. Pharm. Res. 2009; 26: 539-548. http://dx.doi: 10.1007/s11095-008-9769-y.

25 Cheng Y, Zhao L, Li Y and Xu T. Design of biocompatible dendrimers for cancer diagnosis and therapy: current status and future perspectives. Chem. Soc. Rev. 2011; 40: 2673-2703, http://dx.doi: $10.1039 / \mathrm{c} 0 \mathrm{cs} 00097 \mathrm{c}$

26 Duncan R, Izzo L. Dendrimer biocompatibility and toxicity. Adv. Drug Delivery Rev. 2005; 57: 2215-2237. http://dx.doi:10.1016/j. addr.2005.09.019.

27 Bermejo JF, Ortega P, Chonco L, Eritja R, Samaniego R, Mullner M, De Jesus E, De la Mata FJ, Flores JC, Gomez R, and MunozFernan$\operatorname{dez}$ A. Water-soluble carbosilane dendrimers: synthesis biocompatibility and complexation with oligonucleotides; evaluation for medical applications. Chem. Eur. J. 2007; 13: 483-495. http://dx.doi: 10.1002/chem.200600594.

28 Padilla De Jesus OL, Ihre HR, Gagne L, Fréchet JMJ and Szoka Jr FC. Polyester dendritic systems for drug delivery applications: In Vitro and In Vivo evaluation. Bioconjugate Chem. 2002; 13: 453461.http://dx.doi: 10.1021/bc010103m.

29 Kaneshiro TL and Lu ZR. Targeted intracellular codelivery of chemotherapeutics and nucleic acid with a well-defined dendrimerbased nanoglobular carrier. Biomaterials. 2009; 30: 5660-5666. http://dx. doi:10.1016/j.biomaterials.2009.06.026.

30 Wang S, Chen A, Weng L, Chen M, Xie X. Effect of drugloading methods on drug load, encapsulation efficiency and release properties of alginate/poly-L-arginine/chitosan ternary Complex microcapsules. Macromol. Biosci. 2004; 4: 27-30. http://dx.doi: 10.1002/ mabi.200300043.

31 Levy MY, Benita S. Drug release from submicronized o/w emulsion: a new in vitro kinetic evaluation model. Int.J.Pharm.1990; 66: 29-37.

32 Mosinger J, Micka Z. Quantum yields of singlet oxygen of metal complexes of meso- tetrakis(sulphonatophenyl)porphine. J. Photochem. and Photobiol., A. 1997; 107: 77- 82. http://dx.doi.org/10.1016 /S1010-6030(96)04613-8.

Copyright: (c) 2012 M.S. Kumar et al. This is an open-access article distributed under the terms of the Creative Commons Attribution License, which permits unrestricted use, distribution, and reproduction in any medium, provided the original author and source are credited. 